\title{
Committed Moving-Horizon Estimation for Meal Detection and Estimation in Type 1 Diabetes
}

H. Chen ${ }^{1}$, N. Paoletti2 , S.A. Smolka ${ }^{3}$, S. Lin ${ }^{1}$

${ }^{1}$ Department of Electrical and Computer Engineering, Stony Brook University 2Department of Computer Science, Royal Holloway, University of London

${ }^{3}$ Department of Computer Science, Stony Brook University

\section{Meal Detection in the Artificial Pancreas}

\section{Meal Detection and Estimation}

- The artificial pancreas (AP) automates insulin therapy for type 1 diabetes (T1D), using a control algorithm to compute the insulin dose from continuous glucose monitor (CGM) measurements.

- Commercial AP systems require patients to manually announce the amount of carbohydrates ( $\mathrm{CHO})$ at each meal for the AP to deliver adequate insulin.

- Meal announcements are a burden and can be dangerous because they can be incorrect or late.

- Automated meal detection and estimation (MDE) have the potential to replace meal announcements to enable fully closed-loop insulin therapy.
Challenges

- There is a time delay between $\mathrm{CHO}$ ingestion and BG increase. Also, the CGM measures subcutaneous glucose, which is delayed w.r.t. BG. So, MDE needs look-ahead (future CGM measurements) to detect the meal.

- The effect of a meal on BG can be prolonged so MDE also needs history (past CGM measurements).

- MDE must be accurate and timely for effective BG control

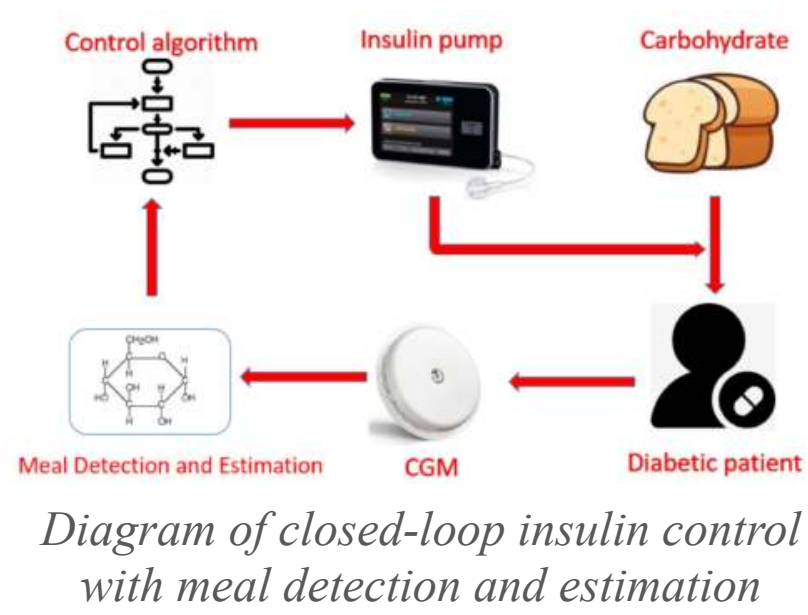

\section{Our idea}

Develop a model-based MDE method that combines meal estimations from CGM made at multiple time instants, thus achieving both look-ahead and history, with guaranteed detection time.

\section{CMHE-based Meal Detection and Estimation}

Moving-Horizon Estimation (MHE)

- MHE uses CGM measurements and a T1D patient model for state estimation, by minimizing the distance between predicted and actual CGM measurements over a moving window of length $N$.

- At any time $t$, the $t$-th MHE instance also estimates the most-likely $\mathrm{CHO}$ disturbances at time $t-N$ to $t-1, \delta_{t-N}^{t}, \ldots, \delta_{t-1}^{t}$.

- With a window of length $N$, we have $\boldsymbol{N}$ possible MHE estimations for the $\mathrm{CHO}$ at time $t$,

$\delta_{t}^{t+1}, \ldots, \delta_{t}^{t+N}$, given by the MHE instances at time $t+1$ to $t+N$.

Which estimation do we commit to?

- Committing only to $\delta_{t}^{t+1}$ lacks look-ahead.

- Committing only to $\delta_{t}^{t+N}$ lacks history: a BG increase for a meal that occurred before $t$ can raise a false alarm.

- We develop a new method, Committed MHE (CMHE), for combining multiple estimations

\section{Committed Moving-Horizon Estimation (CMHE)}

- At time $t, \mathrm{CMHE}$ computes the final $\mathrm{CHO}$ estimation for time $t-V, \Delta_{t-V}$, as a weighted sum of the past $V$ estimations $\delta_{t-V}^{t-V+1}, \ldots, \delta_{t-V}^{t}$

- $V$ is called commitment level, that is, the detection delay.
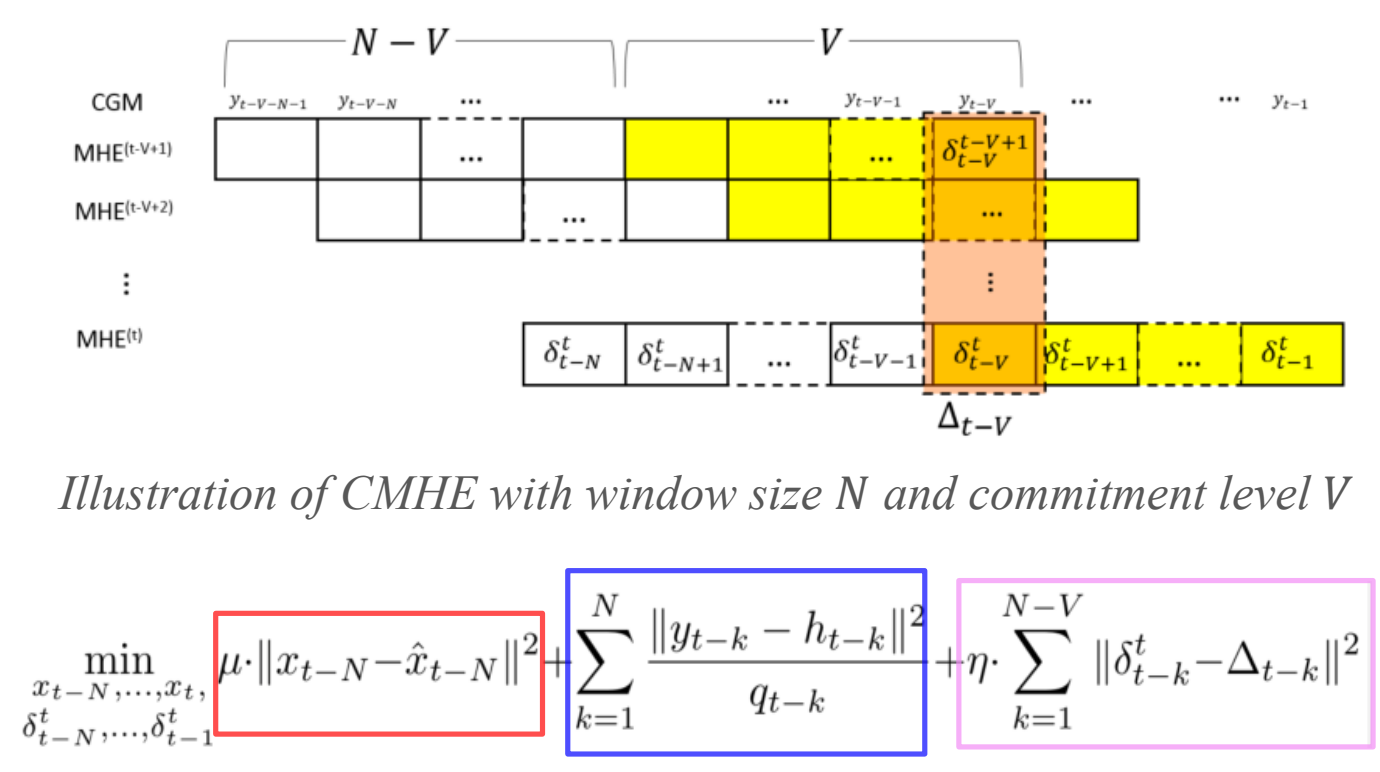

CMHE minimizes the distance between predicted and actual measurements, predicted and estimated states, and

$$
\begin{gathered}
\Delta_{t-V}=\frac{\left(\sum_{i=t-V+1}^{t}(i-t+1)^{b} \cdot \delta_{t-V}^{i}\right)}{\sum_{i=t-V+1}^{t}(i-t+1)^{b}} \\
\begin{array}{c}
\text { CMHE time-based weighting } \\
\text { strategy (tunable to favour history } \\
\text { over look-ahead or vice versa) }
\end{array}
\end{gathered}
$$

Online Detection Algorithm

- It extracts meal onset, duration, and total $\mathrm{CHO}$ amount in real time from CMHE estimations.

- It uses an amplitude threshold $\Delta$ and an edge detector for detecting onset and end of a meal.

\section{Results}

Random Multiple-Meal Experiments

\begin{tabular}{|c|c|c|c|c|c|c|}
\hline & breakfast & snack1 & lunch & snack2 & dinner & snack3 \\
\hline Probability & $100 \%$ & $50 \%$ & $100 \%$ & $50 \%$ & $100 \%$ & $50 \%$ \\
\hline $\begin{array}{l}\text { CHO } \\
\text { amount (g) }\end{array}$ & $40-60$ & $5-25$ & $70-110$ & $5-25$ & $\begin{array}{l}55- \\
75\end{array}$ & $5-15$ \\
\hline $\begin{array}{l}\text { Time of day } \\
\text { (h) }\end{array}$ & $\begin{array}{l}\text { 6:00- } \\
10: 00\end{array}$ & $\begin{array}{l}8: 00- \\
11: 00\end{array}$ & $\begin{array}{l}11: 00- \\
15: 00\end{array}$ & $\begin{array}{l}15: 00- \\
18: 00\end{array}$ & $\begin{array}{l}18: 00- \\
22: 00\end{array}$ & $\begin{array}{l}22: 00- \\
00: 00\end{array}$ \\
\hline \multicolumn{7}{|c|}{ MDE performance } \\
\hline $\begin{array}{l}\text { Onset Devi- } \\
\text { ation (min) }\end{array}$ & 21.97 & 27.00 & 15.28 & 26.42 & 16.32 & 25.08 \\
\hline $\begin{array}{l}\text { CHO Devi- } \\
\text { ation }(\mathrm{g})\end{array}$ & 51.44 & 19.45 & 23.30 & 20.27 & 18.93 & 15.10 \\
\hline $\begin{array}{l}\text { Detection } \\
\text { rate }\end{array}$ & $100 \%$ & $100 \%$ & $100 \%$ & $100 \%$ & $100 \%$ & $66.67 \%$ \\
\hline
\end{tabular}

1) 10 rounds of 3-day simulation, 30 days in total.

2) Large meals and small snacks

3) Random meal $\mathrm{CHO}$ amount and onset

Characteristics and detection/estimation results of each meal

\section{Our Performance}

- At the optimal operating point of the detector, we achieve a $95.38 \%$ daily detection rate.

- For large meals, we have a $100 \%$ detection rate, with an average of 14.9 minutes onset deviation, and $76.6 \% \mathrm{CHO}$ amount estimation accuracy.

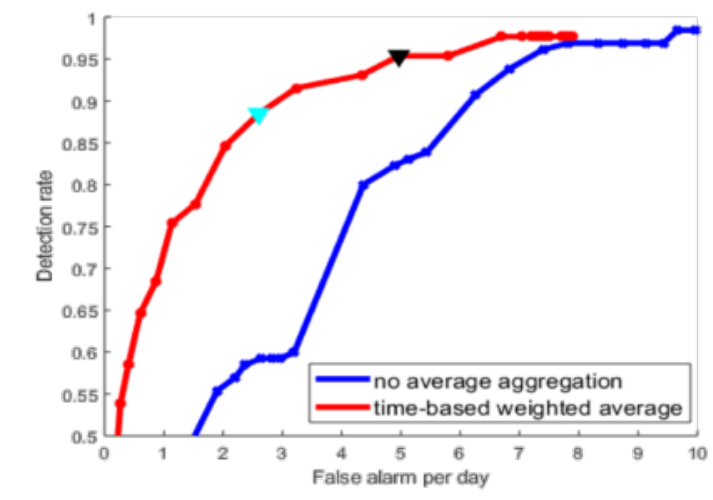

ROC curves of CMHE and MHE obtained with different values for the meal amplitude detection threshold.

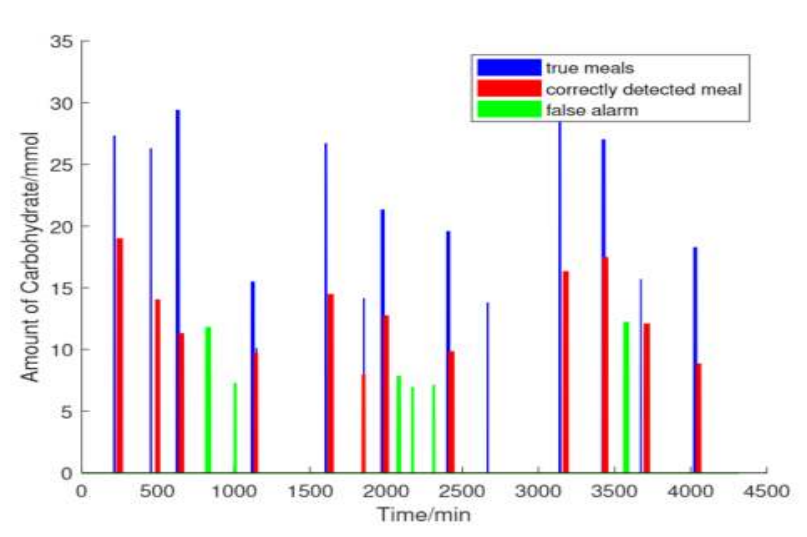

An example of detection results for a 3-day experiment

- Large meals, which cause rapid and large BG increase, are our main priority.

- We achieve $100 \%$ detection rate for all meals but small late-night snacks.

- Detection of small meals requires low detection thresholds, which leads to false alarms. 